\title{
Development of a real-time quantitative PCR method for detection and quantification of Prevotella copri
}

\author{
Phebe Verbrugghe $^{1^{*}}$ (D), Olivier Van Aken ${ }^{2}$, Frida Hållenius ${ }^{1}$ and Anne Nilsson ${ }^{1}$
}

\begin{abstract}
Background: Since its discovery in 2007, the importance of the human gut bacterium Prevotella copri (P. copri) has been widely recognized with its links to diet and health status and potential as next generation probiotic. Therefore, precise, convenient and cost-effective diagnostic tools for the detection and quantification of $P$. copri from clinical and environmental samples are needed.

Results: In this study, a Sybr Green qPCR protocol for P. copri detection and quantification was developed and tested on $P$. copri-spiked murine faeces samples targeting both the $16 \mathrm{~S}$ rRNA gene and $P$. copri genome specific genes. The use of one $16 \mathrm{~S}$ rRNA primer pair and 2 genome specific primer pairs resulted in at least 10x higher specificity and sensitivity than the primer-only PCR currently cited in the literature, reaching a sensitivity of $10^{3}$ CFU/ $\mathrm{mL}$. Furthermore, we showed that the new $16 \mathrm{~S}$ rRNA primer set provided the best balance of detection of a wide range of $P$. copri strains, while avoiding off-target detection of other Prevotella genus species. The quantification of P. copri in human stool samples using the new $16 \mathrm{~S}$ rRNA primers also correlated well with 16S rRNA high throughput MiSeq sequencing data $\left(r^{2}=0.6604, p=0.0074\right)$. The two genome specific primer pairs on the other hand uniquely detect the DSM18205 reference strain, allowing differential detection of indigenous and experimentally administered P. copri populations. Finally, it was shown that SYBR green qPCR mixes have an influence on sensitivity and specificity, with Biorad SsoAdvanced Universal SYBR Green Supermix performing the best under our test conditions of six commercially available SYBR green master mixes.
\end{abstract}

Conclusions: This improved qPCR-based method will allow accurate P. copri identification and quantification. Moreover, this methodology can also be applied to identify other bacterial species in complex samples.

Keywords: Prevotella copri, qPCR, SYBR green

\section{Background}

Human microbiome research has grown exponentially over the last 15 years [1] and many studies have shown an increase or decrease of certain bacterial species in various disease conditions [2]. These reports imply the use of specific bacterial species as potential biomarkers for diet and disease, or as prevention or therapy of diseases. One

\footnotetext{
* Correspondence: vphebe4@yahoo.com

${ }^{1}$ Food Technology, Engineering and Nutrition, Lund University, PO Box 124, 22100 Lund, Sweden

Full list of author information is available at the end of the article
}

common human gut bacterial species that has been associated with diet and disease is Prevotella copri (P. copri). These obligate anaerobic gram-negative rods were first isolated from the human faeces of a Japanese man [3]. They are common in the human gut of communities in under-developed/non-westernised countries [4-6], and their abundance has been linked to healthy vegetable and fiber-rich diets [5]. Regarding its links to health status, decreased levels of $P$. copri were observed in obese women [7] and in subjects with neurodegenerative diseases [8] and childhood atopic dermatitis [9]. In healthy human

C The Author(s). 2021 Open Access This article is licensed under a Creative Commons Attribution 4.0 International License, which permits use, sharing, adaptation, distribution and reproduction in any medium or format, as long as you give appropriate credit to the original author(s) and the source, provide a link to the Creative Commons licence, and indicate if changes were made. The images or other third party material in this article are included in the article's Creative Commons licence, unless indicated otherwise in a credit line to the material. If material is not included in the article's Creative Commons licence and your intended use is not permitted by statutory regulation or exceeds the permitted use, you will need to obtain permission directly from the copyright holder. To view a copy of this licence, visit http://creativecommons.org/licenses/by/4.0/. The Creative Commons Public Domain Dedication waiver (http://creativecommons.org/publicdomain/zero/1.0/) applies to the data made available in this article, unless otherwise stated in a credit line to the data. 
subjects, increased P. copri abundance was correlated with improved glucose tolerance after barley consumption; and in mice, oral administration of live P. copri resulted in improved glucose metabolism [10], suggesting a causal relationship. In line with these findings, $P$. copri has been identified as a candidate for a next generation probiotic to prevent and treat metabolic diseases [11]. On the other hand, increased levels of $P$. copri were reported in patients with new onset untreated rheumatoid arthritis [12] and Irritable Bowel Syndrome [13]. Considering faecal P. copri has been associated with both health benefits and some inflammatory states, it is important to have accurate and rapid methods for testing its abundance.

Until recently, identification of bacterial species from the human gut relied on culture-dependent methods. This lead to a substantial underestimation of biodiversity as the human gut contains bacteria that are either slow growing or unculturable that cannot be detected in this way [14]. The introduction of genetic markers such as the $16 \mathrm{~S}$ rRNA gene, and the development of extensive bacterial genome databases have lessened the need for bacterial cultures, with real-time quantitative PCR (qPCR) now widely used as a technique to identify and quantify target species in a microbial community sample. The $16 \mathrm{~S}$ rRNA gene is present in all bacteria and contains both conserved and hypervariable regions. Universal primers can be designed in the conserved regions while the interspersed hypervariable regions enable discrimination between bacterial families, genera and species.

In our hands, the primer-only PCR currently cited in the literature to detect $P$. copri targeting FimB/Mfa2 family fimbrial subunit (PREVCOP_RS00015) [12] is not sensitive in murine faeces. Indeed, complex biological samples such as faeces and gut content extracts have been shown to complicate accurate analysis [15]. PCR primers and a Taqman probe specific to the V3 region of $P$. copri were recently described by Gray et al. [16] but here we sought to develop a solely primer-based cost effective Sybr Green method.

In this study, 16S rRNA and species-specific primers have been developed to detect and quantify $P$. copri in murine and human faeces, achieving improved $P$. copri quantitative real-time PCR specificity and sensitivity compared to the primers most commonly cited in the literature [12].

\section{Results}

P. copri-specific primer-only PCR currently cited in the literature lacks specificity in complex biological samples The primers most commonly used in the literature to detect $P$. copri [12] targeting FimB/Mfa2 family fimbrial subunit (PREVCOP_RS00015) were initially tested. Though the primers showed good specificity and sensitivity for pure $P$. copri gDNA samples, this was not the case in the presence of murine faeces, where non-specific amplification was observed in the melt curves (multiple peaks indicating different products) at low P. copri concentrations even under stringent PCR conditions (Fig. 1). The standard curves show that the reliable detection threshold is lower when no faeces are present $\left(10^{4} \mathrm{CFU} / \mathrm{mL}\right.$ versus $10^{5} \mathrm{CFU} / \mathrm{mL}$ when faeces are present).

\section{New $P$. copri $16 \mathrm{~S}$ rRNA primers improve specificity}

To increase specificity and sensitivity, a number of primer pairs targeting the $P$. copri $16 \mathrm{~S}$ rRNA non-conserved regions were designed (supplementary Figure 2). As for the original $P$. copri $16 \mathrm{~S}$ rRNA primers described above, there were a number of melt curve peaks in the mixed murine faeces at lower P. copri concentrations indicating binding of the primers to aspecific sequences (Fig. 2). As long amplicon lengths compromise the amplification efficiency when short cycling times are used, only the P.copri_16S_4

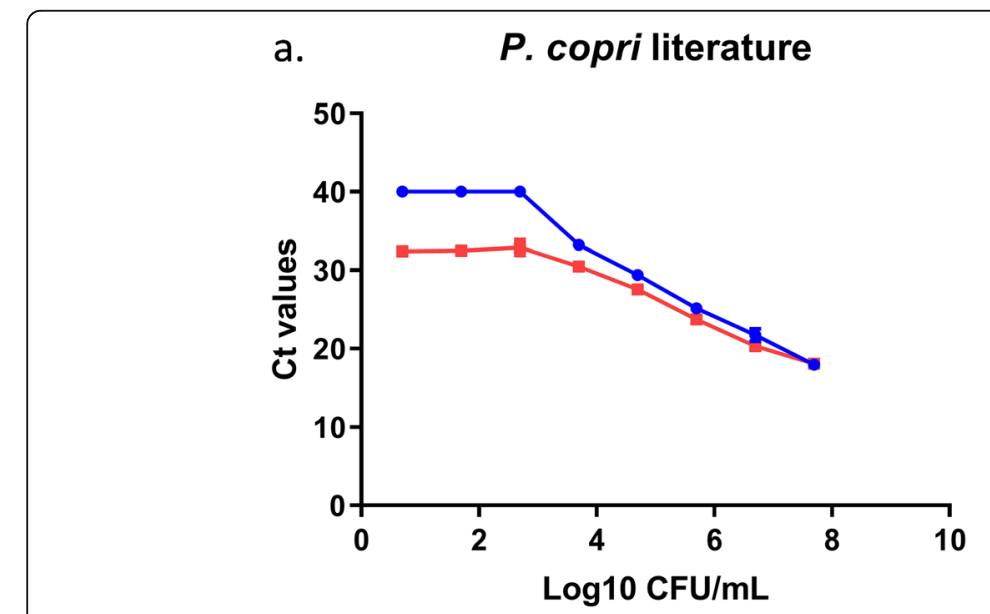

b.
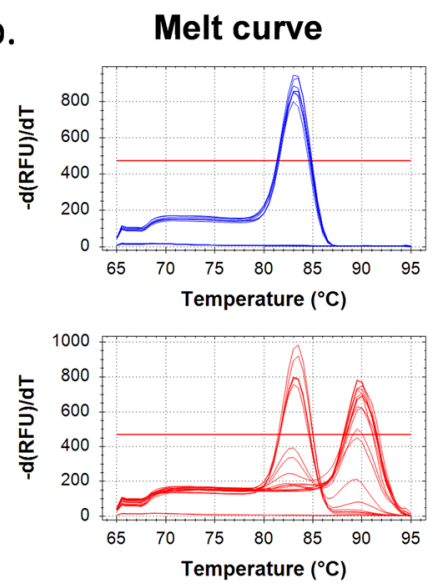

Fig. 1 Performance of $P$. copri genome specific primers from the literature [12] under stringent conditions. Standard curves and melt curves for $P$. copri only (blue) and faeces spiked with P. copri (red) 

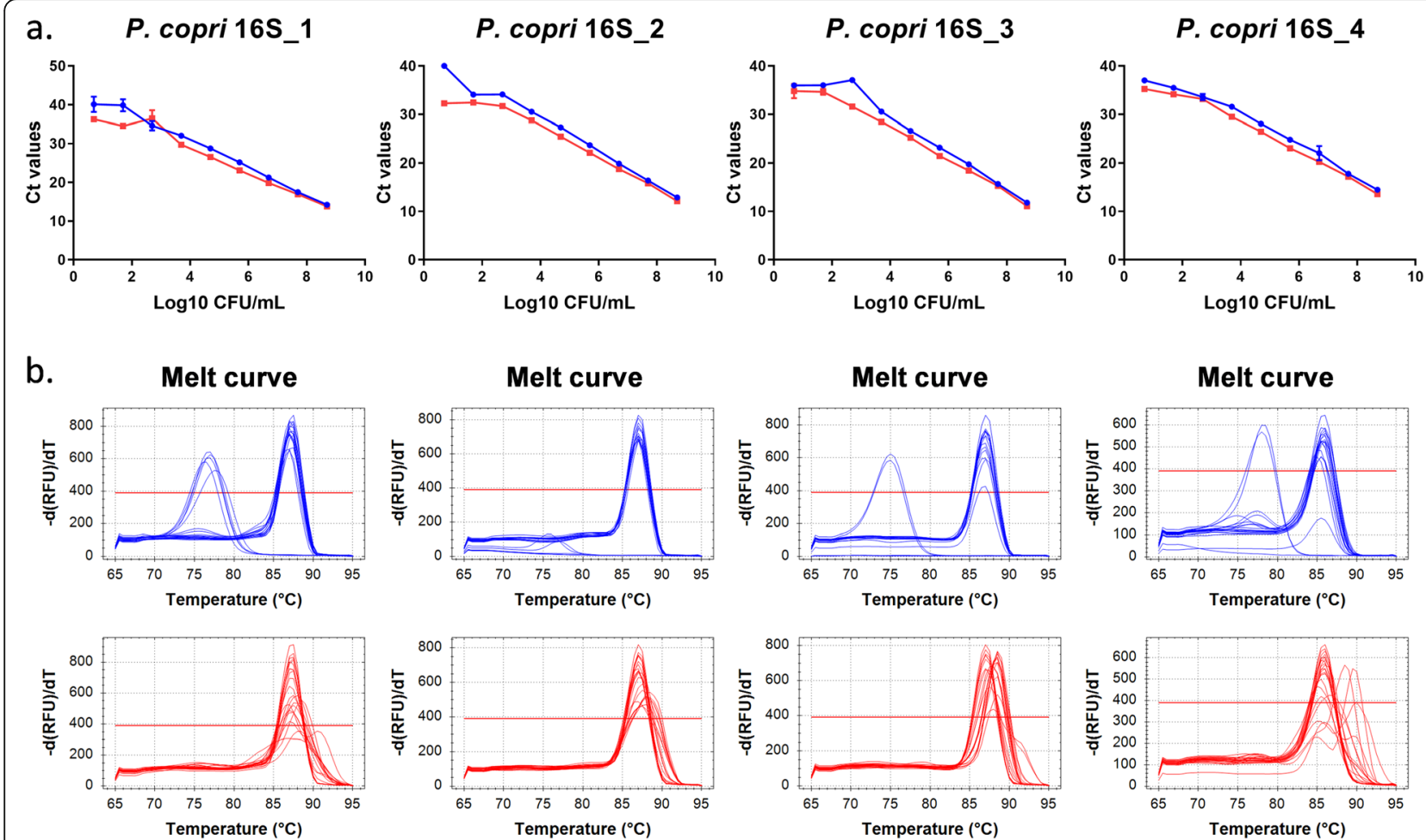

Fig. 2 Performance of newly developed P. copri 16S primers under standard PCR conditions. a Standard curves and b melt curves (blue: P.copri only and red: murine faeces spiked with $P$. copri)

primer pair was tested further under more stringent PCR conditions $(15 \mathrm{~s}$ annealing/extension time instead of $60 \mathrm{~s}$ and increasing annealing/extension temperature from 60 to 62 degrees). Substantial improvement in sensitivity and specificity was observed for P.copri_16S_4 when more stringent PCR conditions were applied. For P.copri_16S_4 primers, the specificity was increased in the lower bacterial concentrations down to $10^{3} \mathrm{CFU} / \mathrm{mL}$ with almost no amplification of aspecific products (all peaks at the same temperature in melt curves) present in the faeces (Fig. 3).

\section{Assessment of $P$. copri genome-specific primers}

To avoid aspecificity due to binding of the very conserved P. copri 16S rRNA gene primers to $16 \mathrm{~S}$ rRNA genes of closely related bacterial species (e.g. other Prevotella species), primers targeting genes unique for the $P$. copri genome were also designed using the published genome sequence of the DSM18205 reference strain (for a list of $P$. copri-specific genes see [17]). Surprisingly however, aspecific product amplification (indicated by additional melt curve peaks at higher temperatures) was still observed in the faeces samples spiked with no or low amounts of bacteria. Figure 4 shows the standard curves generated from serial dilutions of CFU $10^{1}-10^{9} / \mathrm{mL}$ for four $P$. copri specific gene primer sets. Interestingly, $P$. copri genome specific (GS) primers did thus not decrease the background under standard PCR conditions.
Substantial improvement in sensitivity and specificity of the genome specific primers was observed when more stringent PCR conditions were used (15 s annealing/extension time instead of $60 \mathrm{~s}$ and increasing annealing/extension temperature from 60 to 62 degrees). For primer pair P.copri_GS_1 and P.copri_GS_4, the specificity was increased in the lower bacterial concentrations down to $10^{3} \mathrm{CFU} / \mathrm{mL}$ with almost no amplification of aspecific products (all peaks at same temperature in melt curves) present in the faeces (Fig. 5).

\section{The use of different qPCR mastermixes affects sensitivity} and specificity

A number of commercially available qPCR SYBR green mixes was tested for sensitivity and specificity using the P.copri_GS_1 primers (Fig. 6). The specificity, as determined by the number of peaks in the melting curves, was highest for PowerUp SYBR Green Master Mix, FastStart SYBR Green Master Mix, Quantinova SYBR Green PCR kit and SsoAdvanced Universal SYBR Green Supermix (single peak in the melt curves), while amplification of aspecific products could be observed for the qPCRBIO SyGreen Mix and KiCqstart SYBR Green qPCR Ready Mix (multiple peaks in melt curves towards the lower concentrations of bacteria in the presence of faeces). On the other hand, the sensitivity, as determined by the lowest $\mathrm{CFU} / \mathrm{mL}$ concentration before the 


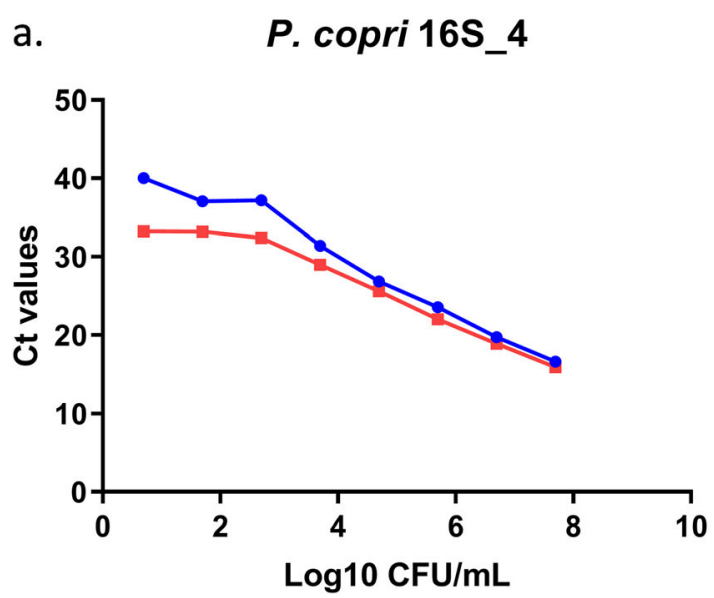

b.
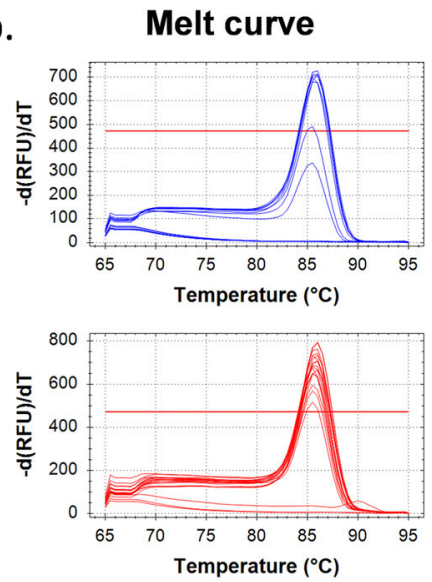

Fig. 3 Performance of newly developed P. copri 165 primers under stringent PCR conditions. a Standard curves and $\mathbf{b}$ melt curves (blue: P. copri only and red: murine faeces gDNA samples spiked with different concentrations of $P$. copri)

standard curves reached a plateau, was highest for SsoAdvanced Universal SYBR Green Supermix, Quantinova SYBR Green PCR Kit, qPCRBIO SyGreen Mix and KiCqstart SYBR Green qPCR Ready Mix $\left(10^{2} \mathrm{CFU} / \mathrm{mL}\right)$, while FastStart SYBR Green Master Mix and PowerUp SYBR Green Master Mix showed the lowest sensitivity $\left(10^{4} \mathrm{CFU} / \mathrm{mL}\right)$. Taken together, SsoAdvanced Universal SYBR Green Supermix, the mix that had been used in the experiments above, resulted in the highest specificity and sensitivity.

\section{Correlation between the relative abundance of $P$. copri} determined by qPCR and 16S rRNA MiSeq sequencing To determine if our qPCR-based quantification method is comparable to $16 \mathrm{~S}$ rRNA profiling by Illumina MiSeq sequencing, we measured relative abundance of $P$. copri

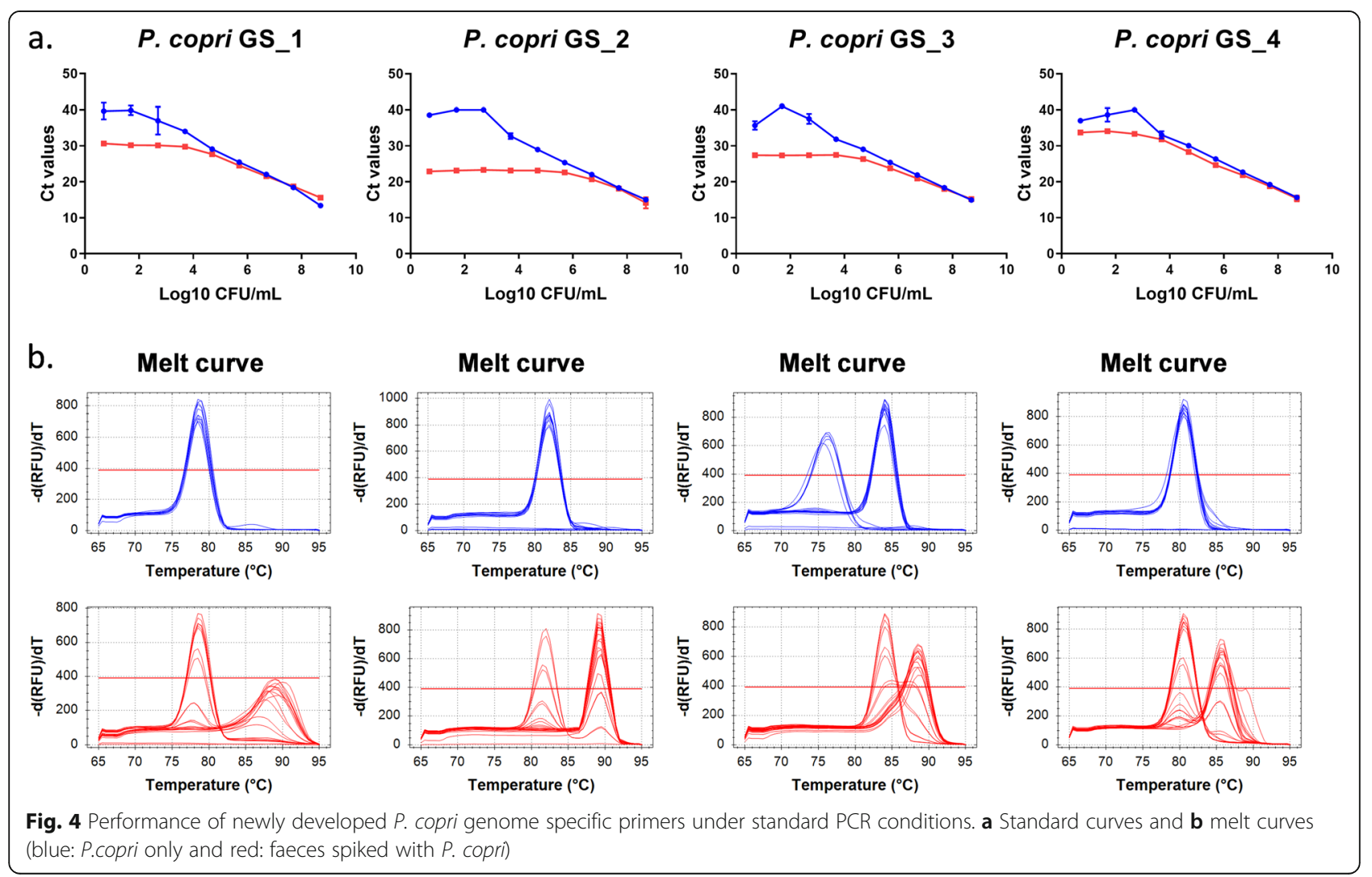




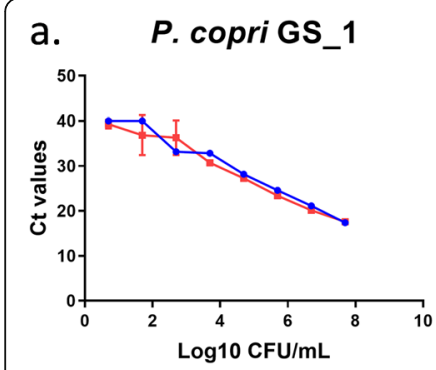

\section{P. copri GS_2}

P. copri GS_3

P. copri GS_4
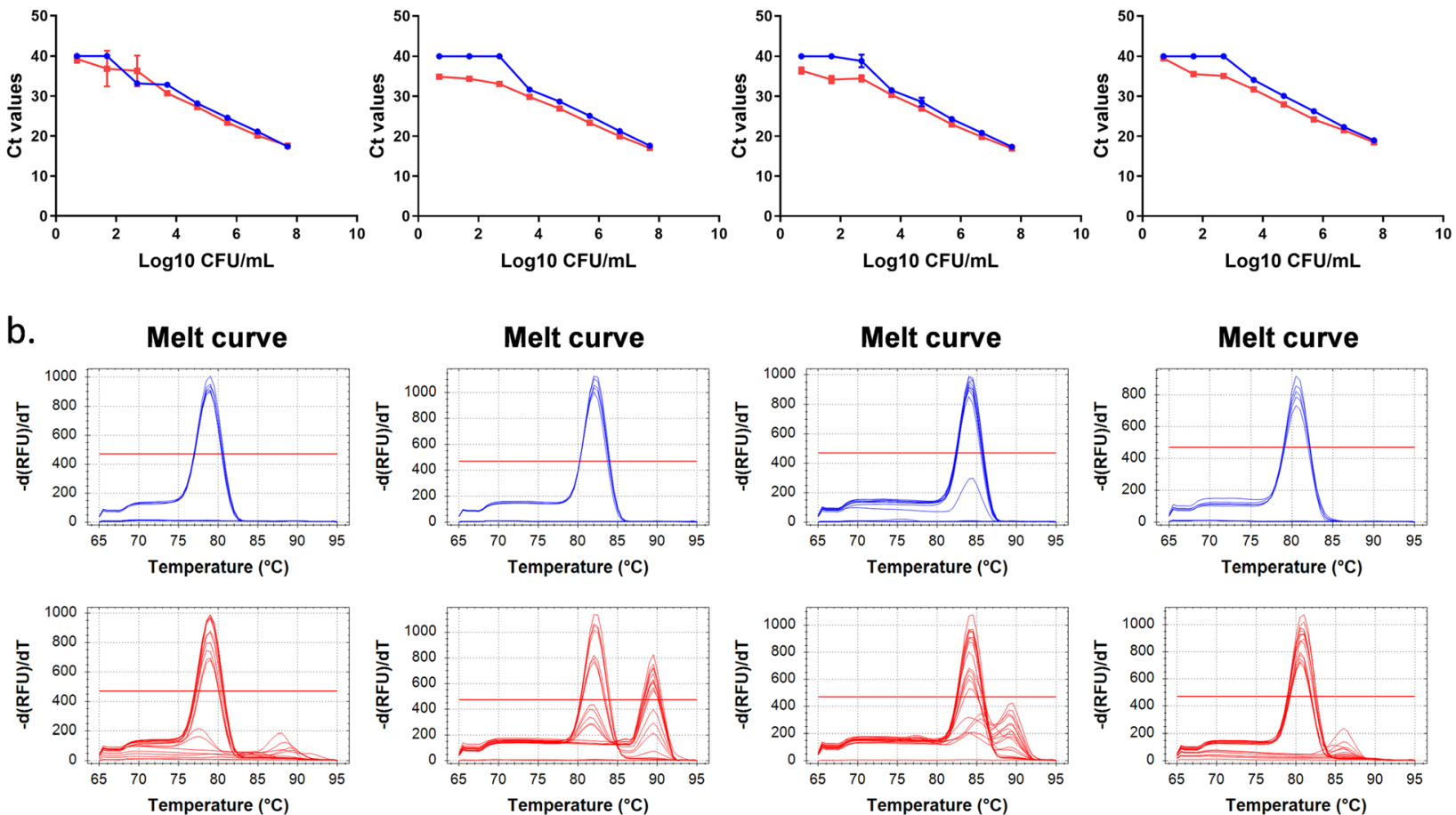

Fig. 5 Performance of newly developed P. copri genome specific primers under stringent PCR conditions. a Standard curves and $\mathbf{b}$ melt curves (blue: P.copri only and red: faeces spiked with P. copri). Primer pairs P.copri_GS_1 and P.copri_GS_4 performed better with the new PCR conditions

in 15 human faeces samples using both methods (Fig. 7). A clear correlation was found between the MiSeq-based method and qPCR using the 16S_4 primers $\left(r^{2}=0.6604\right.$, $p=0.0074)$. In contrast, qPCR using the P.copri_GS_1 and P.copri_GS_4 primers on the human faeces samples yielded no detectable signal (data not shown).

\section{In silico validation of primer binding}

To determine why no signal was detected in the human stool samples using the P.copri_GS_1 and P.copri_GS_4 primers, we analysed in silico binding of the different primer pairs to the sequenced genomes of 114 P. copri strains obtained from the PATRIC database. In agreement, the P.copri_GS_1 and P.copri_GS_4 primers only bound to the reference strain DSM18205, while the P.copri_16S_4 primers bound to 83 of the 114 strains (Supplementary Table 1). We also tested the Scher primers [12], which could only bind to 35 P. copri strains, including the reference strain DSM18205. Another study recently published a Taqman-probe based

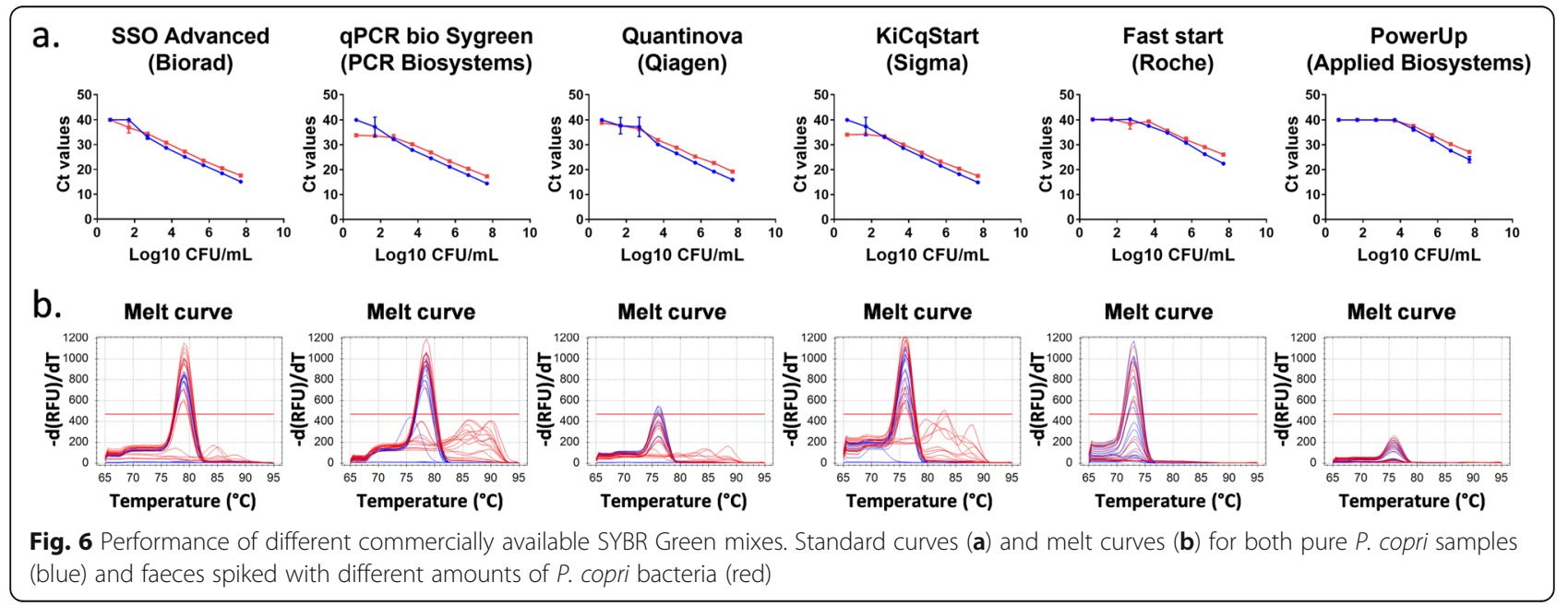



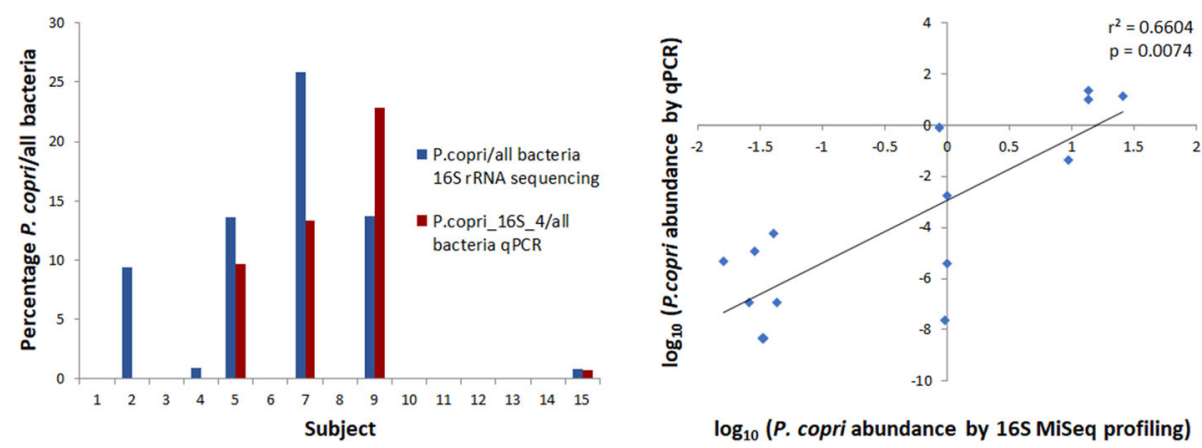

Fig. 7 P. copri abundance in human faeces determined by 16S rRNA gene sequencing and by 16S rRNA qPCR using the P. copri_16S_4 primer set and universal 165 primers (left: bar graph; right: scatter plot). There is a correlation $\left(r^{2}=0.66, p<0.01, n=15\right)$ between the relative abundance of $P$. copri determined by $\mathrm{qPCR}$ and the relative abundance determined by $16 \mathrm{~S}$ rRNA gene sequencing

qPCR method to detect the presence of $P$. copri in a cohort of Australian women [16]. We tested in silico binding of their $16 \mathrm{~S}$ rRNA-based primers in the genomes of the $P$. copri strains, and found they could detect the large majority (107/114) of strains.

To test specificity of our P.copri_16S_4 primers and those developed by Scher et al. and Gray et al. in related species of the Prevotella genus, we analysed in silico binding to the genomes of a range of representative Prevotella species (obtained from the PATRIC database), as represented in the phylogenetic tree (Supplementary Figure 1). While our P.copri_16S_4 primers and the primers developed by Scher et al. yielded no predicted PCR products in any of the tested genomes, the Gray primers yielded nearly identical products of $55 \mathrm{bp}$ in five of the seven non-copri Prevotella species. For the Gray reverse primer, 19 out of 20 bases were direct matches (Supplementary Figure 2), while e.g. 17 out of 19 bases (including the 14 bases at the 3' end) of the Gray forward primers were perfectly matched to the genomes of $P$. jejuni, veroralis, histicola and melanogenica. However, the Gray et al. $14 \mathrm{bp}$ Taqman probe contains two mismatches between P. copri and the Prevotella species mentioned above, so the use of the Gray primers in combination with the TaqMan probe would improve species specificity. Overall, our P.copri_16S_4 primers provide a good balance of detecting a wide range of P.copri strains in a quantitative manner, without off-target detection of other Prevotella genus species while the P.copri_GS_1 and GS_4 primers are highly specific for only the P.copri reference DSM 18205 strain.

No in vitro cross-reactivity with closely related Prevotella species for the newly designed $P$. copri primer sets

As recommended by Balakrishnan et al. [18], the specificity of the primer sets from Scher et al. [12] (Fig. 8a), P.copri_16S_4 (Fig. 8b), P.copri_GS_1 (Fig. 8c) and P.copri_GS_4 (Fig. 8d) was tested by qPCR on DNA of representative species phylogenetically most closely related to P. copri (Supplementary Figures 1 and 3). While the primers from Scher showed cross-reactivity with other Prevotella species such as $P$. veroralis, the newly designed primers specifically and exclusively amplified $P$. copri DNA.

\section{Discussion}

In contrast to traditional culture-dependent methods used in bacterial identification, real-time PCR is fast, cost-effective, quantitative and highly sensitive and is thus increasingly used in clinical diagnostics [19]. Real-time PCR assays, however, are limited by the quality of the primers which must be sensitive enough to target the organism of interest, yet specific enough to exclude all others. The primers for the gut bacterium $P$. copri currently used in the literature [12] work well on pure $P$. copri isolates. Their sensitivity and specificity is however lower in complex samples such as faeces, that contain DNA from e.g. plant, animal and microbial cells present in food, host cells and both living and degraded (micro) organisms, some of which - such as other Prevotella species- are closely related to the species to be identified. In this work, three primer pairs (P.copri_16S_4, P.copri_GS_1 and P.copri_GS_4) were identified that have up to $10 x$ higher specificity and sensitivity in complex biological samples than the primer-only PCR currently cited in the literature [12]. These results highlight the need to test standards in complex samples to ensure accuracy in quantification and eliminate deviations due to other DNA present. Cross-reactivity with the closest related Prevotella species was absent for these newly designed primers while this was not the case for the primer-only PCR currently cited in the literature [12] and recently developed primers by Gray et al [16]. $P$. copri abundance in human faeces determined by qPCR using the newly designed P.copri_16S_4 primers was shown to correlate well with that obtained by 

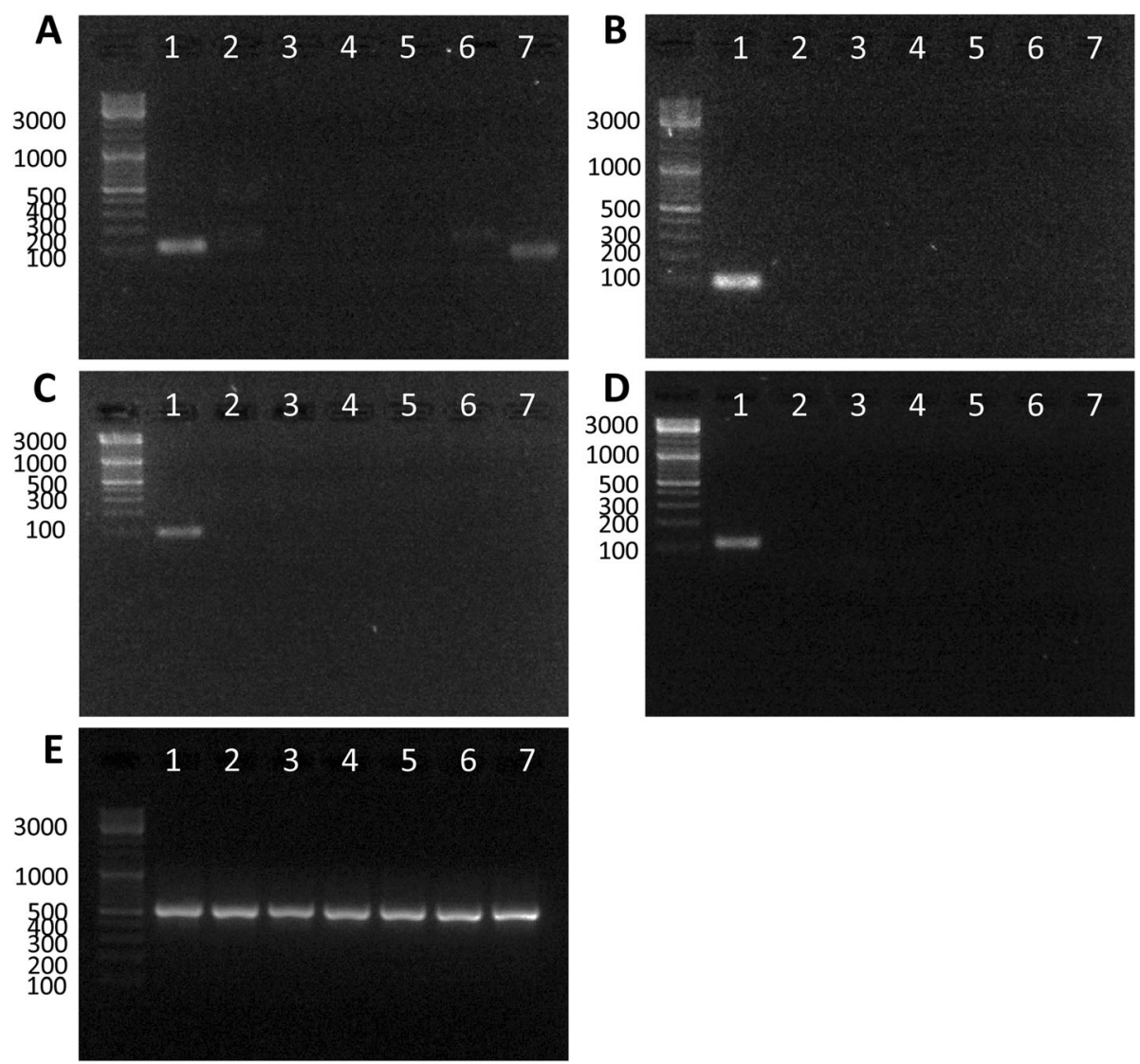

Fig. 8 Specificity of the primer sets validated with related Prevotella species. a primer set P. copri Scher et al. [12]; b primer set P. copri_16S_4: c primer set P. copri_GS_1; d primer set P. copri_GS_4 and e primer set Prevotella genus (Matsuki et al. 2002, [12]) as positive control. Lanes indicate: 1: P. copri; 2: P. salivae; 3: P. paludivivens: 4: P. jejuni; 5: P. melaninogenica; 6: P. histicola; 7: P. veroralis

16S rRNA sequence bacterial profiling, while the P.copri_GS_1 and GS_4 did not yield any products in the human faeces samples. In silico analysis showed that the P.copri_16S_4 primers bound to 83 of 114 P. copri strains (compared to only 35 for the Scher primers) while the primer sets targeting genes unique to (P.copri_GS_1 and GS_4) only bound to the reference strain DSM18205. These data for the non $16 \mathrm{~S}$ gene specific primers are in line with the finding that $P$. copri is one of the most plastic gut colonisers with high subspecies genetic diversity between different subjects [20]. The Gray et al. 16S rRNA primers can potentially detect a wider range of $P$. copri strains, but at the cost of likely detecting non-copri Prevotella species. Therefore, the use of the specific TaqMan probe is most likely required to obtain species specificity. Our 16S rRNA primers have the advantage that they show species specificity without the use of costly TaqMan probes and are compatible with standard SYBR green-based detection methods. The new P.copri_16S_4 primers are thus useful for picking up a wide range of $P$. copri susbspecies (eg. in environmental studies) while the P.copri GS_1 and GS4 primers can be used to specifically detect the DSM18205 reference strain, allowing differential detection and relative quantification of indigenous and administered (eg. to human subjects or mice) P. copri strains. The methodology described here may be useful in the detection of other bacterial species in complex samples. In addition, it was shown that SYBR green PCR mixes have an influence on sensitivity and specificity, with Biorad SsoAdvanced performing the best under our test conditions.

\section{Conclusions}

This qPCR-based method will substantially improve the sensitivity and specificity of P.copri identification and quantification compared to the primer-only qPCR method most commonly cited in the literature [12]. This method also enables the accurate detection of a wider range of $P$. copri subspecies and of $P$. copri DSM18205 specifically. Moreover, the methodology described here can be used as a model to detect and quantify other bacterial species in complex samples. 


\section{Methods}

\section{Cultivation of $P$. copri}

P. copri strain CB7 (DSM 18205, DSMZ GmbH) was cultured on Schaedler agar (Sigma-Aldrich) plates at $37^{\circ} \mathrm{C}$ in an anaerobic $\mathrm{N}_{2} / \mathrm{CO}_{2} / \mathrm{H}_{2}$ atmosphere (85/10/5\%) maintained by a Whitley DG250 workstation. For counting of colony forming units (CFU) serial dilutions of the harvested bacteria were plated on Schaedler agar plates.

\section{Collection of murine faeces}

Faeces were collected from 12 week old healthy male wild type C57BL/6 J mice commercially obtained from Janvier Labs (Marseille, France) and housed in a specific pathogen free animal facility.

\section{gDNA extraction}

P. copri DSM18205 bacteria were harvested from agar plates with sterile loops, washed by suspending in $0.9 \%$ $\mathrm{NaCl}$ and spinning down, resuspended in $0,9 \% \mathrm{NaCl}$ and a fraction was plated out for colony counting. For the pure $P$. copri sample, a volume corresponding to $10^{9}$ bacteria was centrifuged at $3000 \mathrm{rpm}$ and resuspended in lysis buffer. For the $P$. copri-spiked murine faeces samples, faeces were collected from C57BL/6 J mice (Janvier), homogenized using a Qiagen Tissuelyser II and aliquoted. Each aliquot was spiked with $10 \mathrm{uL}$ of up to eight 10 -fold dilutions of $P$. copri bacteria in $0.9 \% \mathrm{NaCl}$ with $10^{1-} 10^{9} \mathrm{CFU} /$ mL. gDNA from bacteria only or faeces spiked with different amounts of bacteria was then prepared according to the manufacturor's instructions using the QIAamp PowerFecal DNA kit (Qiagen, Hilden, Germany). Samples were added to lysis buffer and incubated at $65^{\circ} \mathrm{C}$ for $10 \mathrm{~min}$ and disrupted and homogenized in a Bead Tube containing garnet beads, using a TissueLyser II (Qiagen) prior to DNA extraction. DNA concentrations and purity were measured using a Nanodrop spectrophotometer (Nanodrop Technologies, Wilmington, DE, USA).

\section{Primer design}

$16 \mathrm{~S}$ ribosomal RNA (16S rRNA) gene sequences for the $P$. copri type strain were obtained from NCBI (GenBank accession AB064923). This sequence was aligned with the 7 closest phylogenetically related Prevotella species (as determined by blasting the $P$. copri reference sequence). For the phylogenetic tree, $16 \mathrm{~S}$ rRNA sequences were aligned and a phylogenetic tree was calculated with 1000 bootstraps using ClustalX. The phylogenetic tree was visualised using NJplot (Supplementary Figures 1 and 2). Primers were designed using primer 3 software targeting either the $P$. copri $16 \mathrm{~S}$ rRNA gene or $P$. copri specific genes [17]: glycosyl transferase, group 1 (PREV COP_06806) for primer set P.copri_GS_1, RNA polymerase ECF-type sigma factor (PREVCOP_06715) for primer set P.copri_GS_2, CAAX amino protease family protein (PREVCOP_06538) for primer set P.copri_GS_3 and metalloprotease domain protein, M6 family (PREV COP_04242) for primer set P.copri_GS_4 (for primer details see Table $1[12,21])$. A BLAST [22] search ensured that the primers were not hitting other targets.

\section{Real-time quantitative PCR (qPCR)}

10 -fold serial dilutions in DEPC water were made from $156 \mathrm{ng} / \mu \mathrm{L}$ pure $P$. copri gDNA (equivalent to $10^{9}$ to $10^{1}$ $\mathrm{CFU} / \mathrm{mL}$ ). For gDNA of murine faeces spiked with similar amounts of $P$. copri as for the pure $P$. copri DNA, $20 \mathrm{ng}$ gDNA was used in each qPCR amplification. Samples were run in duplicate on the same plate for the same gene. Detection of the PCR product was carried out by the CFX384 Touch ${ }^{\text {TM }}$ Real-Time PCR system (Biorad, Hercules, CA, USA) using the DNA-binding dye SYBR Green I (SsoAdvanced Universal SYBR Green Supermix unless stated otherwise). The standard 2-step qPCR cycling conditions were as follows: pre-denaturation of $98^{\circ} \mathrm{C}$ for $3 \mathrm{~min}, 40$ cycles of denaturation of $95^{\circ} \mathrm{C}$ for $15 \mathrm{~s}$ and an annealing/extension step at $60^{\circ} \mathrm{C}$ for $1 \mathrm{~min}$. Stringent PCR conditions refer to $15 \mathrm{~s}$ annealing/extension time (instead of $60 \mathrm{~s}$ ) and increasing annealing/extension temperature from 60 to 62 degrees. 'No template' controls (containing DEPC water) were included in each run. The following SYBR Green mastermixes were tested: SsoAdvanced Universal SYBR Green Supermix (Cat No. 1725271, Bio-Rad), qPCRBIO SyGreen Mix (Cat No. PB20.14, PCR Biosystems), PowerUp SYBR Green Master Mix (Cat No. A25741, Applied Biosystems), FastStart SYBR Green Master (Cat. No. 04673 484 001, Roche), KiCqStart SYBR Green qPCR Ready Mix (Cat. No. KCQS00, SigmaAldrich) and QuantiNova SYBR Green PCR Kit (Cat. No. 208059, Qiagen). For cross-reactivity testing, $20 \mathrm{ng}$ DNA of $P$. copri strain CB7 (strain DSM18205), P. salivae (DSM15606), P. paludivivens (DSM17968), P. jejuni (DSM26989), P. melaninogenica (DSM7089), P. histicola (DSM19854) and P. veroralis (DSM19559) (all supplied by DSMZ GmbH) was amplified under stringent PCR conditions as above and was run on a $2 \%$ agarose gel (Sigma-Aldrich) adjacent to the DNA ladder GeneRuler DNA Ladder Mix (Thermofisher scientific).

\section{S RNA sequencing}

Faecal samples were collected in duplicate from 15 subjects (8 male, 7 female) aged 26,9 $\pm 4,3$ years with normal body mass indices (BMI) of $23,9 \pm 3,9 \mathrm{~kg} / \mathrm{m}^{2}$, and immediately stored at $-80^{\circ} \mathrm{C}$ until analysed. Faecal DNA was extracted using the QIAamp Powerfaecal DNA kit (Qiagen), including a bead beating step. Faecal amplicon DNA concentrations were quantified using a Quant-iT dsDNA Assay Kit, High Sensitivity (Life Technologies) using the Fluoroskan ${ }^{\text {TM }}$ Microplate Fluorometer (Life Technologies, Carlsbad, CA, USA). The V4 region of $16 \mathrm{~S}$ rRNA genes was amplified by 
Table 1 Primers used in qPCR studies

\begin{tabular}{|c|c|c|c|c|c|}
\hline Primer name & Sequence & \#nucleotides & $\begin{array}{l}\text { Amplicon } \\
\text { length (bp) }\end{array}$ & Target gene & Reference \\
\hline Universal 16S, F & ACTCCTACGGGAGGCAGCAGT & 21 & 192 & $16 \mathrm{~S}$ rRNA & Scher et al., 2013 [12] \\
\hline Universal 16S, F & ATTACCGCGGCTGCTGGC & 18 & & & \\
\hline P. copri genus $16 \mathrm{~S}, \mathrm{~F}$ & CACRGTAAACGATGGATGCC & 20 & 516 & $16 \mathrm{~S}$ rRNA & Matsuki et al., 2002 \\
\hline P. copri genus $16 \mathrm{~S}, \mathrm{R}$ & GGTCGGGTTGCAGACC & 16 & & & \\
\hline P. copri genome specific, $\mathrm{F}$ & CCGGACTCCTGCCCCTGCAA & 20 & 106 & $\begin{array}{l}\text { FimB/Mfa2 family fimbrial } \\
\text { subunit }\end{array}$ & Scher et al., 2013 [12] \\
\hline P. copri genome specific, $R$ & GTTGCGCCAGGCACTGCGAT & 20 & & PREVCOP_RS00015 & \\
\hline P. copri 16S, 1F & ACATCGAAAGCTTGCTTITG & 20 & 409 & $16 \mathrm{~S}$ rRNA & This publication \\
\hline P. copri 16S, 1R & CAAAAAGCCTCACGAGGCTC & 20 & & AB064923 & \\
\hline P. copri 16S, 2F & ACCACTTGGGGATAACCTTG & 20 & 347 & $16 \mathrm{~S}$ rRNA & This publication \\
\hline P. copri 16S, 2R & TACATGCAAAAAGCCTCACGAGGC & 24 & & AB064923 & \\
\hline P. copri 16S, 3F & TCTCTAGAAGACATCTGAAAGA & 22 & 446 & $16 \mathrm{~S}$ rRNA & This publication \\
\hline P. copri 16S, 3R & CAGTGCAGACGTTGAGCGT & 19 & & AB064923 & \\
\hline P. copri16S, 4F & CGAAAGCTTGCTITTGATGG & 20 & 86 & $16 \mathrm{~S}$ rRNA & This publication \\
\hline P. copri 16S, 4R & CGCAAGGTTATCCCCAAGT & 19 & & AB064923 & \\
\hline P. copri genome specific, $1 \mathrm{~F}$ & TTTGCTGTAGGAGGGGTTG & 20 & 96 & glycosyl transferase, group 1 & This publication \\
\hline P. copri genome specific, $1 \mathrm{R}$ & GGGCTGCATAAAGCAAAGAC & 20 & & PREVCOP_06806 & \\
\hline P. copri genome specific, $2 F$ & AGCCGAGATATCGTGAGTGG & 20 & 137 & $\begin{array}{l}\text { RNA polymerase ECF-type sigma } \\
\text { factor }\end{array}$ & This publication \\
\hline P. copri genome specific, $2 \mathrm{R}$ & TGAACAGCTGTATGCCGAAG & 20 & & PREVCOP_06715 & \\
\hline P. copri genome specific, 3F & AGTTTGTCAATGCCCTCCTG & 20 & 141 & $\begin{array}{l}\text { CAAX amino protease family } \\
\text { protein }\end{array}$ & This publication \\
\hline P. copri genome specific, $3 R$ & CATCGCTCTGAGGCATGATA & 20 & & PREVCOP_06538 & \\
\hline P. copri genome specific, $4 \mathrm{~F}$ & TCGCTGACATGAGCGATAAC & 20 & 109 & $\begin{array}{l}\text { metalloprotease domain protein, } \\
\text { M6 family }\end{array}$ & This publication \\
\hline P. copri genome specific, $4 \mathrm{R}$ & CCGTTGGCACTACCTTCATT & 20 & & PREVCOP_04242 & \\
\hline
\end{tabular}

PCR with forward primer $5^{\prime}$ TCGTCGGCAGCGTCAG ATGTGTATAAGAGACAGGTGCCAGCMGCCGCG GTAA and reverse primer 5' GTCTCGTGGGCTCGGA GATGTGTATAAGAGACAGGGACTACHVG

GGTWTCTAAT [23], and extended with Illumina (Illumina Inc., San Diego, CA, USA) adapter sequences and unique dual indexes to tag each PCR product, according to the $16 \mathrm{~S}$-protocol provided by Illumina. In short, PCRs contained $0.2 \mu \mathrm{M}$ primers, $12.5 \mathrm{ng}$ template DNA and $12.5 \mu \mathrm{L}$ of $2 \times$ KAPA HiFi HotStart Ready Mix kit (KAPA Biosystems, Woburn, MA, USA) in a reaction volume of $25 \mu \mathrm{L}$. Thermal cycling conditions were as follows: initial denaturation at $95^{\circ} \mathrm{C}$ for $3 \mathrm{~min}, 25$ cycles of denaturation at $95^{\circ} \mathrm{C}$ for $30 \mathrm{~s}$, annealing at $55^{\circ} \mathrm{C}$ for 30 $\mathrm{s}$, and extension at $72{ }^{\circ} \mathrm{C}$ for $30 \mathrm{~s}$ and a final step of $72^{\circ} \mathrm{C}$ for $5 \mathrm{~min}$. Purification of the products was carried out with the Agencourt AMPureXP Kit (Beckman Coulter, Miami, FL, USA). Adapters and unique dual indexes were then attached to each sample using a Nextera XT index kit (Illumina), after which a second cleanup step was performed using the AMPureXP Kit
(Beckman Coulter). The size of the PCR amplicons was verified using $0.1 \%$ agarose gel electrophoresis. No visible bands were observed for the negative extraction controls. $4 \mathrm{pM}$ of the amplicons and 5\% of PhiX control v3 (internal control) were collected into a single tube. Paired-end sequencing with a read length of $2 \times 300 \mathrm{bp}$ was performed on a Miseq Instrument (Illumina) using a Miseq v2 reagent kit (Illumina). The sequence analysis, finally, was carried out using the free software package Quantitative Insights into Microbial Ecology (QIIME).

\section{Supplementary Information}

The online version contains supplementary material available at https://doi. org/10.1186/s12866-020-02063-4.

Additional file 1: Figure S1. Phylogenetic tree of the species closest related to Prevotella copri.

Additional file 2: Figure S2. CLUSTAL O (1.2.4) multiple sequence alignment. Primers are indicated by arrows.

Additional file 3: Figure S3. Specificity of the primer sets validated with related Prevotella species (cf. Fig. 8) with inverted alternative exposures. A: primer set P.copri Scher et al. [12]; B: primer set P. 
copri_16S_4: C: primer set P.copri_GS 1: D: primer set P.copri_GS 4 and E: primer set Prevotella genus (Matsuki et al. 2002, [12]) as positive control. Lanes indicate: 1: P. copri; 2: P. salivae; 3: P. paludivivens: 4: P. jejuni; 5: P. melaninogenica; 6: P. histicola; 7: P. veroralis.

Additional file 4: Table S1. In silico analysis of primer binding to $114 P$. copri strains obtained from the PATRIC database. Columns contain the following information: genome name, PATRIC genome ID, whether both forward and reverse primers bind to strain (for P.copri_GS_1,

P.copri_GS_4, P.copri_16S_4, P. copri primers used in Scher et al. [12] and Gray et al. [16]); size of genome of $P$. copri strain and number of contigs.

\section{Abbreviations}

P. copri: Prevotella copri; CFU: Colony forming units; QPCR: Quantitative Polymerase Chain Reaction; 16S rRNA: 165 ribosomal RNA; gDNA: Genomic DNA

\section{Acknowledgements}

We would like to thank Olex Fedkiv for help with the culturing of Prevotella copri, Ling Cao for technical help with MiSeq sequencing and Stephen Burleigh for assistance with the in silico analysis of the $16 \mathrm{~s}$ rRNA gene sequencing data.

\section{Authors' contributions}

$\mathrm{PV}, \mathrm{FH}$ and $\mathrm{AN}$ were involved in the conception of the study. PV and OV were involved in the acquisition, analysis or interpretation of the data. PV, $\mathrm{OV}, \mathrm{FH}$ and $\mathrm{AN}$ were involved in the writing of the manuscript. All authors have read and approved the manuscript.

\section{Funding}

This work has been performed within Swelife (2017-03575), a strategic innovation program financed by Vinnova, and was also supported by grants by the Royal Physiographic Society of Lund (39893) and the Direktör Albert Påhlsson Foundation (FB2018-0297). These funding bodies had no role in the design of the study and collection, analysis, and interpretation of data nor in writing the manuscript. Open Access funding provided by Lund University.

\section{Availability of data and materials}

The datasets used and analysed in the current study are available from the corresponding author on reasonable request.

\section{Ethics approval and consent to participate}

The mouse faeces collection was approved by the local Malmö-Lund Ethical Review Committee for Animal Experimentation and conducted in accordance with the European Community regulation concerning the protection of experimental animals (2010/63/EU).

Faeces collection of human subjects was approved by the Regional Ethical Review Board in Lund, Sweden (Reference 2018/41). The study was conducted according to the guidelines described in the Declaration of Helsinki and was registered at ClinicalTrials.gov (NCT03423381).

\section{Consent for publication}

Not applicable.

\section{Competing interests}

The authors declare that they have no competing interests.

\section{Author details}

${ }^{1}$ Food Technology, Engineering and Nutrition, Lund University, PO Box 124, 22100 Lund, Sweden. ${ }^{2}$ Department of Biology, Lund University, Lund University Plant Sciences, Sölvegatan 35, 22362 Lund, Sweden.

\section{Received: 28 January 2020 Accepted: 7 December 2020}

Published online: 11 January 2021

\section{References}

1. Jones S. Trends in microbiome research. Nat Biotechnol. 2013;31:277 https:// doi.org/10.1038/nbt.2546.

2. Wu H, Tremaroli V, Backhed F. Linking microbiota to human diseases: a systems biology perspective. Trends Endocrinol Metab. 2015;26:758-70 https://doi.org/10.1016/j.tem.2015.09.011.
3. Hayashi H, Shibata K, Sakamoto M, Tomita S, Benno Y. Prevotella copri sp. nov. and Prevotella stercorea sp. nov., isolated from human faeces. Int J Syst Evol Microbiol. 2007;57:941-6 https://doi.org/10.1099/ijs.0.64778-0.

4. De Filippo C, et al. Impact of diet in shaping gut microbiota revealed by a comparative study in children from Europe and rural Africa. Proc Natl Acad Sci U S A. 2010;107:14691-6 https://doi.org/10.1073/pnas.1005963107.

5. Schnorr SL, et al. Gut microbiome of the Hadza hunter-gatherers. Nat Commun. 2014;5:3654 https://doi.org/10.1038/ncomms4654.

6. Martinez I, et al. The gut microbiota of rural papua new guineans: composition, diversity patterns, and ecological processes. Cell Rep. 2015;11: 527-38 https://doi.org/10.1016/j.celrep.2015.03.049.

7. Dugas LR, et al. Decreased microbial co-occurrence network stability and SCFA receptor level correlates with obesity in African-origin women. Sci Rep. 2018:8:17135 https://doi.org/10.1038/s41598-018-35230-9.

8. Gerhardt S, Mohajeri MH. Changes of colonic bacterial composition in Parkinson's disease and other neurodegenerative diseases. Nutrients. 2018; 10 https://doi.org/10.3390/nu10060708.

9. Mahdavinia $\mathrm{M}$, et al. Effects of diet on the childhood gut microbiome and its implications for atopic dermatitis. J Allergy Clin Immunol. 2019;143:16361637.e1635 https://doi.org/10.1016/j.jaci.2018.11.034.

10. Kovatcheva-Datchary $P$, et al. Dietary fiber-induced improvement in glucose metabolism is associated with increased abundance of Prevotella. Cell Metab. 2015;22:971-82 https://doi.org/10.1016/j.cmet.2015.10.001.

11. Chang CJ, et al. Next generation probiotics in disease amelioration. J Food Drug Anal. 2019;27:615-22 https://doi.org/10.1016/j.jfda.2018.12.011.

12. Scher JU, et al. Expansion of intestinal Prevotella copri correlates with enhanced susceptibility to arthritis. Elife. 2013;2:e01202 https://doi.org/10. 7554/eLife.01202.

13. Su T, et al. Altered intestinal microbiota with increased abundance of Prevotella is associated with high risk of diarrhea-predominant irritable bowel syndrome. Gastroenterol Res Pract. 2018;2018:6961783 https://doi. org/10.1155/2018/6961783.

14. Hiergeist A, Glasner J, Reischl U, Gessner A. Analyses of intestinal microbiota: culture versus sequencing. ILAR J. 2015;56:228-40 https://doi.org/10.1093/ ilar/ilv017.

15. Brankatschk R, Bodenhausen N, Zeyer J, Burgmann H. Simple absolute quantification method correcting for quantitative PCR efficiency variations for microbial community samples. Appl Environ Microbiol. 2012;78:4481-9 https://doi.org/10.1128/AEM.07878-11.

16. Gray $L$, et al. Rapid PCR identification of Prevotella copri in an Australian cohort of pregnant women. J Dev Orig Health Dis. 2020;11:228-34 https:// doi.org/10.1017/S2040174419000849.

17. Gupta VK, Chaudhari NM, Iskepalli S, Dutta C. Divergences in gene repertoire among the reference Prevotella genomes derived from distinct body sites of human. BMC Genomics. 2015;16:153 https://doi.org/10.1186/ s12864-015-1350-6.

18. Balakrishnan B, et al. Development of a real-time PCR method for quantification of Prevotella histicola from the gut. Anaerobe. 2017:48:37-41 https://doi.org/10.1016/j.anaerobe.2017.06.019.

19. Espy MJ, et al. Real-time PCR in clinical microbiology: applications for routine laboratory testing. Clin Microbiol Rev. 2006;19:165-256 https://doi. org/10.1128/CMR.19.1.165-256.2006.

20. Truong DT, Tett A, Pasolli E, Huttenhower C, Segata N. Microbial strain-level population structure and genetic diversity from metagenomes. Genome Res. 2017;27:626-38 https://doi.org/10.1101/gr.216242.116.

21. Matsuki T, Watanabe K, Fujimoto J, Miyamoto Y, Takada T, Matsumoto K, Oyaizu H, Tanaka R. Development of 165 rRNA-Gene-Targeted GroupSpecific Primers for the Detection and Identification of Predominant Bacteria in Human Feces. Appl Environ Microbiol. 2002:68(11):5445-51.

22. Altschul SF, Gish W, Miller W, Myers EW, Lipman DJ. Basic local alignment search tool. J Mol Biol. 1990;215:403-10 https://doi.org/10.1016/S00222836(05)80360-2.

23. Kozich JJ, Westcott SL, Baxter NT, Highlander SK, Schloss PD. Development of a dual-index sequencing strategy and curation pipeline for analyzing amplicon sequence data on the MiSeq Illumina sequencing platform. Appl Environ Microbiol. 2013;79:5112-20 https://doi.org/10.1128/AEM.01043-13.

\section{Publisher's Note}

Springer Nature remains neutral with regard to jurisdictional claims in published maps and institutional affiliations. 